\title{
Diversidad y valor de importancia para la conservación de la vegetación natural. Parque Nacional Lihué Calel (Argentina) ${ }^{1}$
}

\author{
Alicia María CAMPO \\ Consejo Nacional de Investigaciones Científicas y Técnicas \\ Departamento de Geografía y Turismo \\ Universidad Nacional del Sur \\ amcampo@uns.edu.ar \\ Valeria Soledad DuvaL \\ Comisión de Investigaciones Científicas \\ Departamento de Geografía y Turismo \\ Universidad Nacional del Sur \\ valeria.duval@uns.edu.ar
}

Recibido: 29 de noviembre del 2013

Enviado a evaluar: 7 de enero del 2014

Aceptado: 24 de junio del 2014

\section{RESUMEN}

La vegetación es un recurso natural clave para el equilibrio del ecosistema por lo que es necesario disponer de información cuantitativa sobre sus características y distribución. El objetivo del presente trabajo fue determinar la diversidad y el valor de importancia de la vegetación natural en el Parque Nacional Lihué Calel a través de índices cuantitativos con el propósito de contribuir a su conservación. Se calcularon parámetros tales como frecuencia, dominancia y densidad. Sus valores relativos permitieron conocer el índice de valor de importancia de cada especie de Cottam y Curtis. Además se obtuvieron el índice de riqueza específica de Margalef y Menhinick, de dominancia de Simpson, de equidad de Shannon-Wiener y de Pielou para conocer la diversidad de la vegetación. Como resultado se identificaron 33 especies pertenecientes principalmente al estrato arbustivo y herbáceo. Las familias representativas fueron Larrea y Poaceae. Los valores de los índices mostraron que existe una gran cantidad de especies en el área, que la comunidad no posee especies dominantes y por lo tanto la diversidad es mayor; que es una formación equitativa y que todas las especies son abundantes. Estas conclusiones permitieron comprender la necesidad de conservar la vegetación natural del área.

Palabras clave: vegetación, índices de diversidad, índice de valor de importancia, Parque Nacional Lihué Calel.

\footnotetext{
${ }^{1}$ Trabajo realizado en el marco del proyecto Geografía Física aplicada al estudio de la interacción sociedad-naturaleza. Problemáticas a diferentes escalas témporo-espaciales. Dirigido por la Dra. Alicia María Campo. Secretaría de Ciencia y Tecnología, Universidad Nacional del Sur.
} 


\title{
Diversity and value of importance of vegetation for their conservation. Parque Nacional Lihué Calel (Argentina)
}

\begin{abstract}
The vegetation is a natural resource for the balance of the ecosystem so it is necessary to have quantitative information on their characteristics and distribution. The purpose of this was to determine the diversity and value of importance of natural vegetation in the Parque Nacional Lihué Calel through quantitative indices in order to contribute to their conservation. Quantitative parameters were calculated such as frequency, dominance and density. Their relative values allowed to know the importance value index of each species. Also obtained the index of Margalef species richness, the Simpson dominance index and equity index Shannon-Wiener and Pielou for to know the diversity of vegetation. As a result there werw identified 33 species belonging mainly to shrub and herbaceous. The representative families were Larrea and Poaceae. The values of the indices showed that there is a lot of species in the area, the community has no dominant species and therefore diversity is greater, the formation is equitable and all species are abundant. These conclusions allowed us to understand the need to preserve the natural vegetation of the area.
\end{abstract}

Key words: vegetation, diversity indices, importance value index, Parque Nacional Lihué Calel.

\section{La diversité et le valeur d'importance de la végétation pour son conservation. Parque Nacional Lihué Calel (Argentine)}

\section{RESUMÈ}

La végétation est la composante clé pour l'équilibre de l'écosystème. Par conséquent, il est nécessaire de recueillir des informations quantitatives sur ses caractéristiques et sa distribution. L'objectif de ce travail a été de déterminer la diversité et l'indice de valeur d'importance de la végétation dans le Parque Nacional Lihué Calel en utilisant des indices quantitatifs afin de contribuer à sa conservation. Des divers paramètres quantitatifs ont été calculés, tels que la fréquence, la domination et de la densité. La valeur relative de chaque paramètre a permis de connaître la valeur d'importance de chaque espèce. Également, cette étude utilise l'indice de richesse des espèces Margalef, l'indice de dominance Simpson et l'indice d'équité Shannon-Wiener et Pielou pour evaluer le diversité de la végétation dans la région. Trente-trois espèces ont été observées, notamment du type arbustif et herbacé. Les familles le plus représentatives sont Larrea et Poaceae.

Les valeurs des indices ont montré qu'il ya beaucoup d'espèces dans la région, la communauté n'a pas les espèces dominantes et donc la diversité est plus grande, ce qui est une formation équitable et que toutes les espèces sont abondantes. Ces résultats nous ont permis de comprendre la nécessité de préserver la végétation naturelle de la région.

Mots-clés: végétation, indices de diversité, indice de valeur d'importance, Parque Nacional Lihué Calel. 


\section{INTRODUCCIÓN}

La conservación de los ecosistemas en áreas protegidas comprende la protección de la composición, estructura y funcionamiento de los elementos que constituyen la biodiversidad. $\mathrm{Su}$ protección es una problemática compleja que requiere de un entendimiento profundo de la relación ambiente-sociedad en espacios geográficos concretos. La variedad y cantidad de los tipos de vegetación son indicadores relevantes en el análisis de la biodiversidad de un ecosistema para su conservación (Luebert y Becerra, 1998).

Los inventarios describen la estructura y función de la vegetación para su aplicación en el uso y manejo de la misma (Álvarez et al., 2006). La caracterización de sus propiedades fisonómicas permite el reconocimiento de la complejidad estructural presente mientras que su representación mediante fórmulas resume la información en un solo valor. También es posible realizar comparaciones entre la diversidad de distintos hábitats o la diversidad de un mismo hábitat a través del tiempo. Los índices cuantitativos muestran la relevancia de su conservación en áreas protegidas (Suárez y Vischi, 1997).

Zabalza et al. (1989) realizaron un relevamiento fitosociológico en el cual contabilizaron la existencia de 13 comunidades florísticas. Como síntesis cartografiaron las grandes áreas de vegetación dividiéndolas en crestas y áreas con roca desnuda, zonas de pendientes, depresiones, lomadas, piedemonte y mosaico de vegetación.

Entre los antecedentes de trabajo en el área se destaca Mazzola et al. (2008) quienes realizaron un estudio sobre la incidencia del gradiente altitudinal en la vegetación de las Sierras de Lihué Calel. Esta investigación se realizó en el Cerro Fortaleza en la que se identificaron las especies vegetales y su distribución asociadas al factor topográfico. El método elegido fue el de transecta teniendo en cuenta la altura y exposición de las laderas. Se realizó un análisis de las condiciones climáticas con el fin de contrastar la variabilidad de los datos en los distintos parámetros. Se comprobó que la exposición de la ladera juega un papel determinante sobre la distribución de la vegetación, siendo la ladera norte la de mayor presencia de especies vegetales.

La biogeografía y la ecología del paisaje han aportado los principales fundamentos teóricos para el diseño de las áreas naturales protegidas. En particular, la biogeografía cuantitativa es un conjunto de técnicas de análisis útiles en la descripción y análisis biogeográficos. Analiza unidades geográficas entendidas como unidades mínimas de análisis en la cual los índices de diversidad son fórmulas básicas utilizadas para caracterizar a una región en términos de su biota (Murguía y Rojas, 2001). La información cuantitativa de la vegetación en las áreas protegidas contribuye a su conservación. Es por ello que el objetivo principal fue determinar la diversidad y el valor de importancia de la vegetación en el Parque Nacional Lihué Calel a través de índices cuantitativos. La originalidad radica en que es el primer trabajo que analiza la diversidad de vegetación en esta área protegida mediante índices cuantitativos. 


\section{2. ÁREA DE ESTUDIO}

El Parque Nacional Lihué Calel es un área protegida localizada en el centro-sur de la provincia de La Pampa, Argentina (Figura 1). Se creó en el año 1977 y abarca 32.000 ha. Tiene como objetivo principal conservar el sistema serrano homónimo y especies de fauna y flora endémicas. También se protegen los yacimientos arqueológicos y sitios históricos que forman parte del patrimonio cultural de la provincia. Dentro del parque se diferencian tres tipos de ambientes: el serrano, el jarillal y el salitral. Teniendo en cuenta las categorías de manejo de la Unión Internacional para la Conservación de la Naturaleza (2013) esta área protegida es un Parque Nacional (categoría II), Reserva Natural Estricta (categoría I) y Reserva Nacional (categoría IV).

Por otro lado, Morello et al. (2012) determinaron que esta área se halla inserta en la ecorregión Monte de Llanuras y Mesetas. La misma se extiende al este de la Cordillera de los Andes, desde la provincia de Mendoza incluyendo Neuquén y La Pampa, hasta la costa del océano Atlántico en la provincia de Río Negro y nordeste de Chubut. La distribución de la vegetación está controlada por la topografía y el tipo de suelo (Matteucci, 2012). En la zona de sierras existen cuatro formaciones vegetales: arbustal abierto perennifolio de Larrea divaricata con gramíneas bajas que ocupan el $80 \%$ de la superficie, el bosque abierto caducifolio de Prosopis flexuosa con Acantholippia seriphioides, el arbustal de Larrea cuneifolia y el pastizal de gramíneas bajas y vegetación halófila (Cano, 2004).

El clima dominante es el semiárido con una temperatura media anual de $16,2{ }^{\circ} \mathrm{Cy}$ una precipitación media total de $416 \mathrm{~mm}$. Los veranos e inviernos térmicos están bien diferenciados y las dos estaciones intermedias poseen valores similares. Las temperaturas máximas absolutas se registran en el mes de diciembre superando los 35 ${ }^{\circ} \mathrm{C}$ y las mínimas absolutas en junio y agosto, siendo cercanas a $-5^{\circ} \mathrm{C}$. La mayor cantidad de precipitaciones se registran en los meses de marzo, febrero y enero. En el balance hídrico del periodo 1995-2010 (Figura 2) se reconoce un déficit de 434,44 $\mathrm{mm}$ durante todo el año. Esto significa que la evapotranspiración potencial o necesidad de agua supera significativamente a la precipitación. 
Figura 1. Localización del Parque Nacional Lihué Calel.

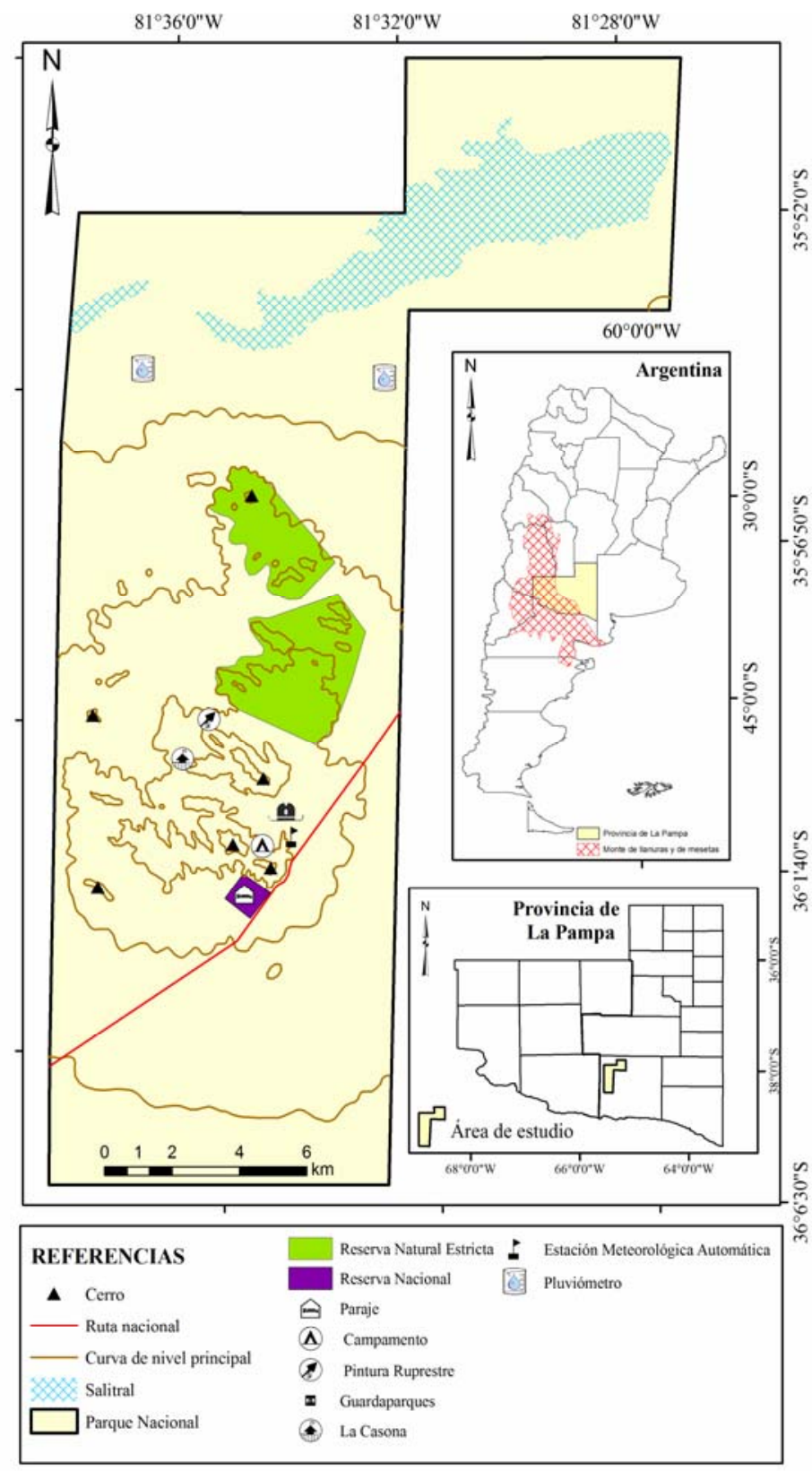

Fuente: Elaborado por Valeria S. Duval sobre la base de cartas topográficas IGM, escala 1:100.000, 2013. 
Figura 2. Balance hídrico de Parque Nacional Lihué Calel. Período 1995-2010.

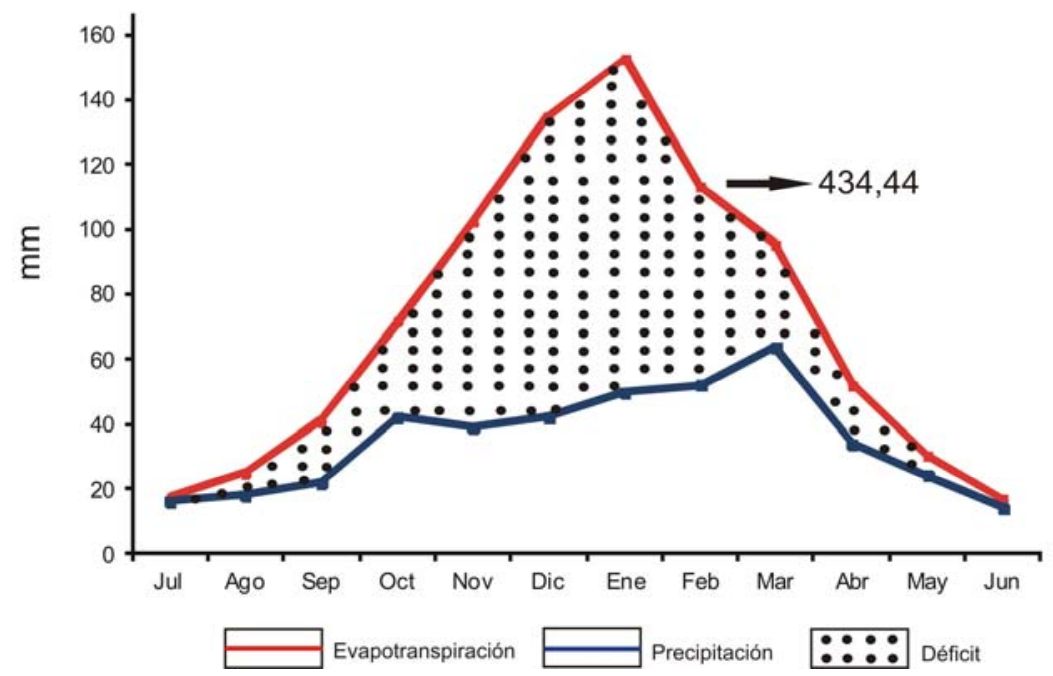

Fuente: Elaborado por Valeria S. Duval sobre los datos proporcionados por el Parque Nacional Lihué Calel, 2013.

\section{METODOLOGÍA}

El estudio florístico se realizó sobre la base de 10 parcelas de $100 \mathrm{~m}^{2}$ en el sector sureste del Parque Nacional Lihué Calel, siendo la muestra total de 0,1 ha. Este valor se obtuvo de la aplicación del área mínima o curva de área-especie. En el relevamiento de los individuos vegetales se tuvieron en cuenta las formas de vida fanerófitos y caméfitos de Raunkiaer (1934). Las primeras poseen yemas perennes que se encuentran por encima de $\operatorname{los} 25 \mathrm{~cm}$ y son leñosas mientras que las segundas tienen sus yemas en la superficie del suelo hasta los $25 \mathrm{~cm}$ de altura. Ambas son especies permanentes, es decir sobreviven a condiciones térmicas y pluviométricas desfavorables. En cada una de las parcelas se registró el número de especies, el número de individuos y la cobertura. Luego se calcularon la densidad, la dominancia y la frecuencia, tanto absoluta como relativa. Se obtuvo el índice de riqueza específica de Margalef; índice de diversidad de Menhinick, el índice de dominancia y diversidad de Simpson, el índice de equidad de Shannon-Wiener y Pielou y el Índice de Valor de Importancia (IVI) de cada especie.

La diversidad es el número de especies en una unidad de área que se mide a través de dos métodos: la riqueza específica basada en la cantidad de especies presentes y la estructura que mide la distribución proporcional del valor de importancia (Figura 3). Este último se clasifica en la dominancia y en equidad de la comunidad (Moreno, 
2001). Existen distintos tipos de diversidad: la local o diversidad $\alpha$ (alfa), la diferenciación de la diversidad entre áreas o diversidad $\beta$ (beta) y la diversidad $\gamma$ (gamma) que reúne a las dos anteriores. En este estudio se utiliza la diversidad alfa que es la riqueza de especies de una comunidad particular a la que se considera homogénea (Smith, 2001).

Figura 3. Métodos para evaluar la diversidad alfa.

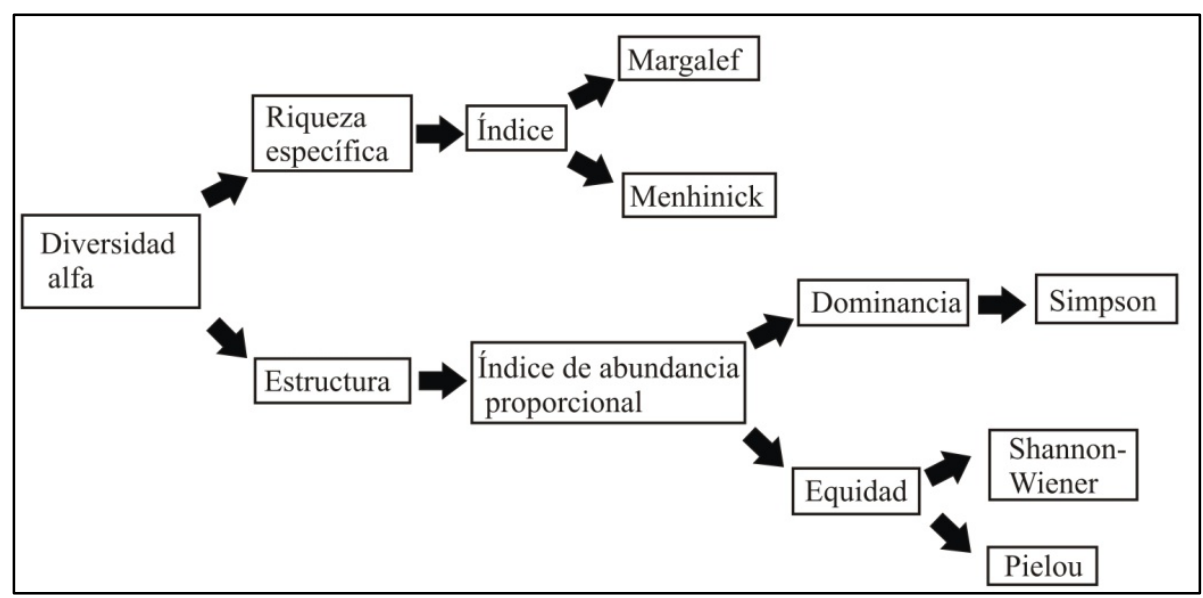

Fuente: Elaborado por Valeria S. Duval sobre la base de Moreno, 2001.

El índice de Margalef $\left(\mathrm{D}_{M g}\right)$ [1] es una forma sencilla de medir la biodiversidad ya que proporciona datos de riqueza de especies de la vegetación. Mide el número de especies por número de individuos especificados o la cantidad de especies por área en una muestra (Margalef, 1969). Su fórmula es:

$$
\mathrm{D}_{\mathrm{Mg}}=\frac{\mathrm{S}-1}{\ln N}
$$

donde:

$\mathrm{S}=$ número de especies.

$N=$ número total de individuos.

El índice de diversidad de Menhinick $\left(\mathrm{D}_{M n}\right)[2]$ al igual que el índice de Margalef se basa en la relación entre el número de especies y el número total de individuos observados, que aumenta al aumentar el tamaño de la muestra.

$$
\mathrm{D}_{M n}=\frac{\mathrm{S}}{\sqrt{N}}
$$


El índice de dominancia de Simpson ( $\mathrm{D}_{S i}$ ) [3] considera la probabilidad que dos individuos de la población seleccionados al azar sean de la misma especie. Indica la relación existente entre riqueza o número de especies y la abundancia o número de individuos por especie. Su expresión es:

$$
\mathrm{D}_{S i}=\sum_{i=1}^{S} p_{i}^{2}
$$

donde:

$p i$ igual a la proporción entre ni y $N$

ni: número de individuos de la especie $i$.

El índice de Shannon- Wiener $\left(H^{\prime}\right)$ [4] tiene en cuenta la riqueza de especies y su abundancia. Este índice relaciona el número de especies con la proporción de individuos pertenecientes a cada una de ellas presente en la muestra. Además mide la uniformidad de la distribución de los indixiduos entre las especies. Su fórmula es:

$$
H^{*}=-\sum p t \cdot \ln p t
$$

El índice de Pielou $\left(J^{\prime}\right)$ [5] se expresa como el grado de uniformidad en la distribución de individuos entre especies. Se puede medir comparando la diversidad observada en una comunidad contra la diversidad máxima posible de una comunidad hipotética con el mismo número de especies (Moreno, 2001).

$$
J^{\prime}=\frac{H^{\prime}}{H^{\prime} \max }
$$

donde:

$H^{\prime}{ }_{\text {máx. }}$ : es el logaritmo natural de $\mathrm{S}$

El índice de valor de importancia define cuáles de las especies presentes contribuyen en el carácter y estructura de un ecosistema (Cottam y Curtis, 1956). Este valor se obtiene mediante la sumatoria de la frecuencia relativa [6], la densidad relativa [7] y la dominancia relativa [8].

Frecuencia relativa $=$ Frecuencia de la $\mathrm{sp} \times 100$

[6]

Frecuencia de todas las spp

Densidad relativa $=\frac{\text { Núm. de individuos de la especie }}{\text { Núm. total de individuos) }} \times 100$

[7]

Dominancia relativa $=\underline{\text { Dominancia de la } \mathrm{sp}} \times 100$

[8]

Dominancia de todas las spp 


\section{RESULTADOS}

\subsection{CARACTERIZACIÓN DE LA VEGETACIÓN MUESTREADA}

La vegetación predominante en la provincia fitogeográfica del monte es el matorral o la estepa arbustiva xerofítica y la comunidad clímax es el jarillal (Figura 4) (Passera et al., 2010). Este es un arbustal denso compuesto por dos estratos verticales de vegetación definidos. El estrato arbustivo está representado por Larrea divaricata. En el Parque Nacional Lihué Calel se observa el predominio de la familia de la Zygophyllaceae (190 individuos), Verbenaceae (162) y Fabaceae (156); especies que registraron mayor cantidad de individuos. Con respecto al biotopo, el arbustivo es el más representativo mientras que el arbóreo es el que posee menor porcentaje de presencia (Figura 5). La mayor parte de la vegetación característica de la provincia fitogeográfica del monte es perenne.

Figura 4. Vegetación del jarillal en el Parque Nacional Lihué Calel.

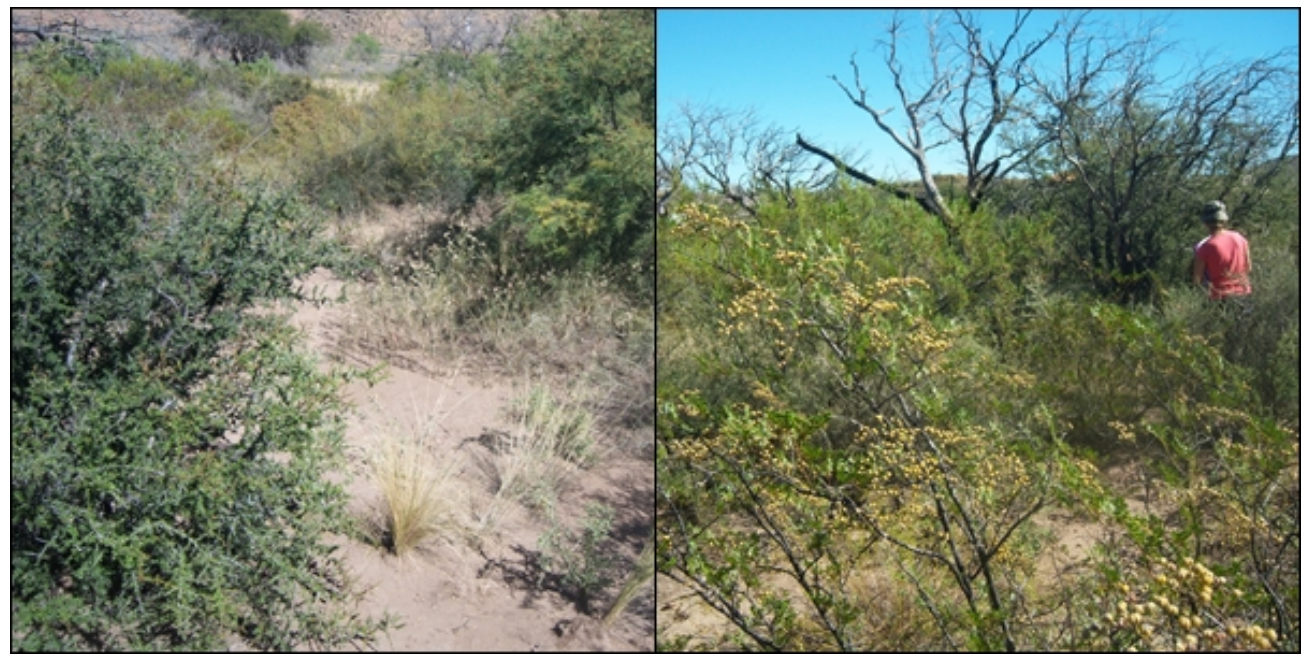

Fuente: fotografías tomadas por Valeria S. Duval, 2012. 
Figura 5. Número de individuos por familia y biotopo de la vegetación.

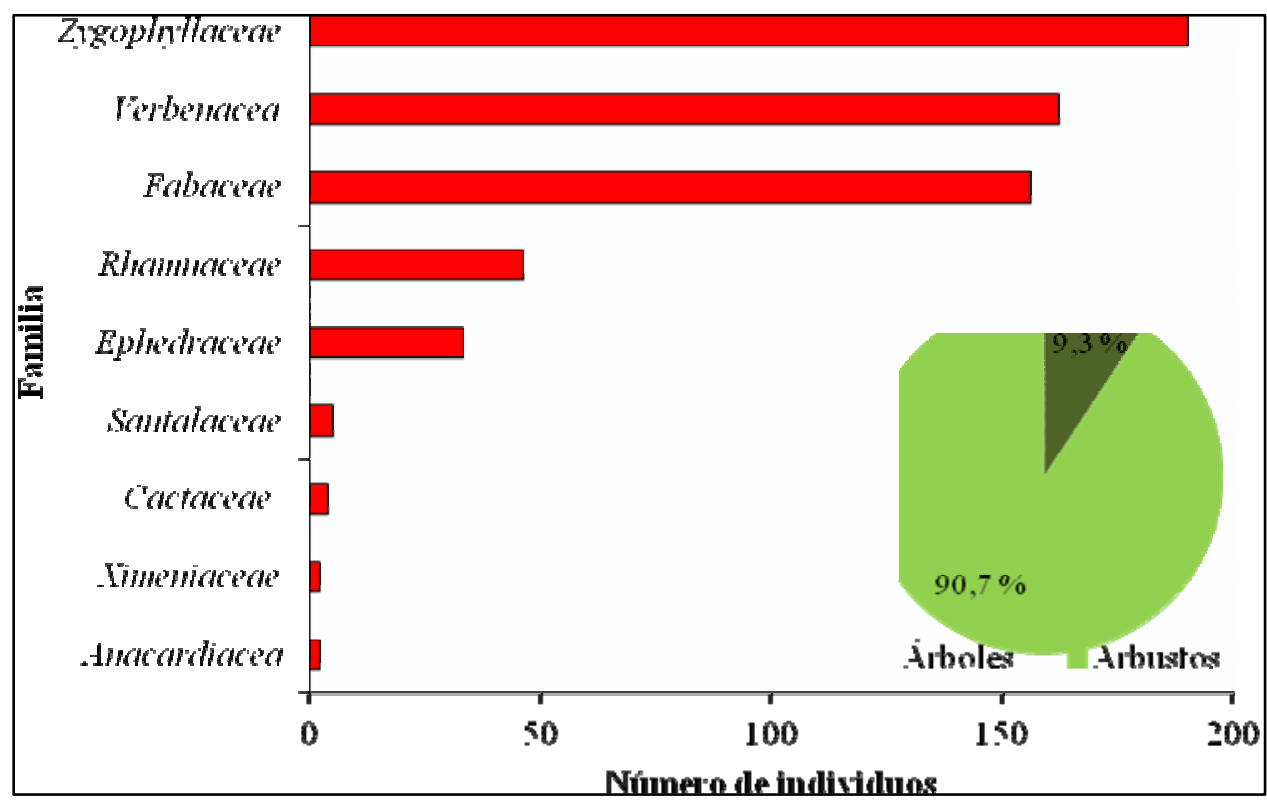

Fuente: Elaborado por Valeria S. Duval sobre la base de trabajo de campo, 2013.

La Tabla 1 muestra las especies relevadas en las parcelas, su nombre vulgar, el número de individuos de cada especie y el biotipo. Baccharis salicifolia $y$ Glandularia hookeriana son las dos especies que posee mayor cantidad de individuos con respecto a las otras. Las especies que tienen mayor cobertura absoluta son Larrea divaricata y la Larrea nítida. 
Tabla 1. Caracterización de la vegetación en las parcelas.

\begin{tabular}{|c|c|c|c|}
\hline Especies & Nombre vulgar & $\begin{array}{|lr|}\mathrm{N}^{\circ} & \mathrm{de} \\
\text { individuos }\end{array}$ & Biotipo \\
\hline $\begin{array}{l}\text { Aloysia } \\
\text { gratissima }\end{array}$ & $\begin{array}{l}\text { Azahar del } \\
\text { monte }\end{array}$ & 46 & Arbusto \\
\hline $\begin{array}{l}\text { Baccharis } \\
\text { salicifolia }\end{array}$ & Chilca & 158 & Arbusto \\
\hline Baccharis ulicina & Yerba de oveja & 27 & Arbusto \\
\hline $\begin{array}{l}\text { Caesalpinia } \\
\text { gilliesii }\end{array}$ & Barba de chivo & 19 & Arbusto \\
\hline Cassia aphylla & Pichicanilla & 25 & Arbusto \\
\hline Cereus aethiops & Penca & 3 & Arbusto \\
\hline $\begin{array}{l}\text { Chuquiraga } \\
\text { erinacea }\end{array}$ & Chilladora & 2 & Arbusto \\
\hline $\begin{array}{l}\text { Condalia } \\
\text { microphylla }\end{array}$ & Piquillín & 46 & Arbusto \\
\hline Ephedra triandra & Tramontana & 33 & Arbusto \\
\hline $\begin{array}{l}\text { Geoffroea } \\
\text { decorticans }\end{array}$ & Chañar & 68 & Árbol \\
\hline $\begin{array}{l}\text { Glandularia } \\
\text { hookeriana }\end{array}$ & \begin{tabular}{|l|} 
Margarita \\
amarga
\end{tabular} & 116 & Arbusto \\
\hline Hyalis argentea & Olivillo & 5 & Arbusto \\
\hline $\begin{array}{l}\text { Jodina } \\
\text { rhombifolia }\end{array}$ & Sombra de toro & 5 & Árbol \\
\hline Larrea cuneifolia & Jarilla macho & 28 & Arbusto \\
\hline Larrea divaricata & Jarilla & 71 & Arbusto \\
\hline Larrea nítida & \begin{tabular}{|l|} 
Jarilla crespa \\
\end{tabular} & 91 & Arbusto \\
\hline $\begin{array}{l}\text { Opuntia } \\
\text { puelchana }\end{array}$ & Puel & 1 & Arbusto \\
\hline $\begin{array}{l}\text { Prosopidastrum } \\
\text { globosum }\end{array}$ & Man & 38 & Arbusto \\
\hline $\begin{array}{l}\text { Prosopis } \\
\text { alpataco }\end{array}$ & Аाра & 5 & Arbusto \\
\hline $\begin{array}{l}\text { Prosopis } \\
\text { caldenia }\end{array}$ & Caldén & 1 & Árbol \\
\hline $\begin{array}{l}\text { Schinus } \\
\text { fasciculatus }\end{array}$ & Molle negro & 2 & Arbusto \\
\hline $\begin{array}{l}\text { Ximenia } \\
\text { americana }\end{array}$ & Albaricoque & 6 & Arbusto \\
\hline
\end{tabular}

Fuente: Elaboración por Valeria S. Duval sobre la base de trabajo de campo, 2013. 


\section{2 ÍNDICES DE DIVERSIDAD Y VALOR DE IMPORTANCIA}

La fórmula [1] demuestra la alta o baja diversidad del ecosistema en relación con la vegetación. Los valores inferiores a 2 son zonas de baja diversidad y los valores superiores a 5 son indicativos de alta biodiversidad (Margaleff, 1995). De la aplicación de la fórmula se obtuvo un resultado de 7,24 lo cual demuestra que el Parque Nacional Lihué Calel es una zona que tiene una significativa cantidad de especies vegetales. La aplicación de la fórmula [2] es de 0,78 por lo que verifica que la riqueza dentro de la muestra analizada es alta.

$$
\begin{aligned}
& \mathrm{D}_{M n}=\frac{22}{\sqrt{796}}=0,78 \\
& \mathrm{D}_{M g}=\frac{22-1}{\ln 796}=7,24
\end{aligned}
$$

El índice de dominancia de Simpson muestra resultados entre 0 a 1 en el cual los valores cercanos a 1 explican la dominancia de una especie por sobre las demás; son ecosistemas más homogéneos. La aplicación de la fórmula [3] dio como resultado 0,1038 por lo tanto no muestra especies dominantes. Si bien Baccharis salicifolia es la especie que cuenta con mayor cantidad de individuos hay otras como Glandularia hookeriana, Larrea nítida y Larrea divaricata que también están representadas de la misma forma. La diversidad puede calcularse como $\mathrm{D}=1-\lambda$ debido a que el valor de dominancia es inverso a la equidad. El valor que se obtuvo de la aplicación de esta fórmula fue 0,8962 , lo cual demuestra que es una formación vegetal muy diversa.

El índice de Shannon-Wiener adquiere valores entre 0 cuando hay una sola especie y el logaritmo neperiano de $\mathrm{S}$ cuando todas las especies están representadas por el mismo número de individuos (Moreno, 2001). El resultado de la aplicación de dicho índice [4] es de 2,51 por lo tanto demuestra que esta comunidad es equitativa. En la Tabla 2 se representa los valores correspondientes al índice de Simpson y el de Shannon-Wiener.

El índice de equidad de Pielou posee valores que pueden variar de 0 a 1 siendo cercanos a 1 los que corresponden a situaciones donde todas las especies son igualmente abundantes. El resultado de la aplicación de este índice [5] en las parcelas de vegetación es igual a 0,86 . Para la fórmula se utilizó $\mathrm{H}^{\prime}$ previamente obtenida en el índice de Shannon-Wiener.

$$
J^{\prime}=\frac{2,51}{\ln 796}=0,86
$$


Tabla 2. Resultados del índice de Simpson y Shannon Wiener para cada especie.

\begin{tabular}{|l|r|r|}
\hline \multicolumn{1}{|c|}{ Especies } & $\begin{array}{c}\text { Índice de } \\
\text { dominancia } \\
\text { de Simpson }\end{array}$ & $\begin{array}{c}\text { Índice de } \\
\text { Shannon- } \\
\text { Wiener }\end{array}$ \\
\hline Aloysia gratissima & 0,003 & $-0,165$ \\
\hline Baccharis salicifolia & 0,039 & $-0,321$ \\
\hline Baccharis ulicina & 0,001 & $-0,115$ \\
\hline Caesalpinia gilliesii & 0,001 & $-0,089$ \\
\hline Cassia aphylla & 0,001 & $-0,109$ \\
\hline Cereus aethiops & 0,000 & $-0,021$ \\
\hline Chuquiraga erinacea & 0,000 & $-0,015$ \\
\hline Condalia microphylla & 0,003 & $-0,165$ \\
\hline Ephedra trianda & 0,002 & $-0,132$ \\
\hline Geoffroea decorticans & 0,007 & $-0,210$ \\
\hline Glandularia hookeriana & 0,021 & $-0,281$ \\
\hline Hyalis argentea & 0,000 & $-0,032$ \\
\hline Jodina rhombifolia & 0,000 & $-0,032$ \\
\hline Larrea cuneifolia & 0,001 & $-0,118$ \\
\hline Larrea divaricata & 0,008 & $-0,216$ \\
\hline Larrea nítida & 0,013 & $-0,248$ \\
\hline Opuntia puelchana & 0,000 & $-0,008$ \\
\hline Prosopidastrum globosum & 0,002 & $-0,145$ \\
\hline Prosopis alpataco & 0,000 & $-0,032$ \\
\hline Prosopis caldenia & 0,000 & $-0,008$ \\
\hline Schinus fasciculatus & 0,000 & $-0,015$ \\
\hline Ximena americana & 0,000 & $-0,037$ \\
\hline Total & 0,104 & 2,513 \\
\hline
\end{tabular}

Fuente: Elaboración por Valeria S. Duval, 2013. 


\section{3 ÍNDICE DE VALOR DE IMPORTANCIA}

En la Tabla 3 se ordenaron las especies en función al IVI de mayor a menor. La misma muestra también los resultados de frecuencia relativa [6], densidad relativa [7] y dominancia relativa para cada especie [8]. Aloysia gratissima, Caesalpinia gilliesii, Condalia microphylla y Larrea divaricata fueron las especies que registraron mayor frecuencia relativa.

Tabla 3. Frecuencia, densidad y dominancia relativa e IVI

\begin{tabular}{|l|r|r|r|r|}
\hline Especies & $\begin{array}{c}\text { Frecuencia } \\
\text { relativa \% }\end{array}$ & $\begin{array}{c}\text { Densidad relativa } \\
\%\end{array}$ & $\begin{array}{c}\text { Dominancia } \\
\text { relativa \% }\end{array}$ & \multicolumn{1}{c|}{ IVI } \\
\hline Larrea divaricata & 8,57 & 8,92 & 17,47 & 34,96 \\
\hline Larrea nítida & 5,71 & 11,43 & 16,56 & 33,71 \\
\hline $\begin{array}{l}\text { Glandularia } \\
\text { hookeriana }\end{array}$ & 4,29 & 14,57 & 11,54 & 30,4 \\
\hline Baccharis salicifolia & 5,71 & 19,85 & 4,23 & 29,8 \\
\hline Aloysia gratissima & 11,43 & 5,78 & 9,85 & 27,1 \\
\hline Geoffroea decorticans & 8,57 & 8,54 & 6,92 & 24 \\
\hline Condalia microphylla & 8,57 & 5,78 & 7,39 & 21,7 \\
\hline Caesalpinia gilliesii & 10,00 & 2,39 & 8,14 & 20,5 \\
\hline Prosopidastrum \\
globosum & & & & \\
\hline Larrea cuneifolia & 7,14 & 4,77 & 1,42 & 13,3 \\
\hline Baccharis ulicina & 4,29 & 3,52 & 5,10 & 12,9 \\
\hline Ephedra trianda & 2,71 & 3,39 & 0,87 & 9,9 \\
\hline Cassia aphylla & 1,43 & 4,15 & 1,24 & 8,2 \\
\hline Jodina rhombifolia & 1,43 & 3,14 & 1,07 & 5,6 \\
\hline Prosopis alpataco & 1,43 & 0,63 & 2,70 & 4,7 \\
\hline Ximena americana & 1,43 & 0,63 & 1,93 & 3,9 \\
\hline Schinus fasciculatus & 2,86 & 0,75 & 1,28 & 3,5 \\
\hline Cereus aethiops & 2,86 & 0,25 & 0,32 & 3,4 \\
\hline Prosopis caldenia & 1,43 & 0,38 & 0,13 & 3,4 \\
\hline Hyalis argentea & 1,43 & 0,13 & 1,01 & 2,6 \\
\hline Chuquiraga erinacea & 1,43 & 0,63 & 0,34 & 2,4 \\
\hline Opuntia puelchana & 1,43 & 0,25 & 0,40 & 2,1 \\
\hline Total & 100 & 0,13 & 0,11 & 1,7 \\
\hline & & 100 & 100 & 300 \\
\hline
\end{tabular}

Fuente: Elaboración por Valeria S. Duval, 2013. 
Esto se deriva de la presencia u ocurrencia de estas especies en la mayoría de las muestras relevadas. Del total de las 10 parcelas, la primera especie se observó en 8 parcelas, la segunda se registró en 7 y la tercera y cuarta en 6 parcelas. Este parámetro está condicionado por el número y tamaño de los individuos dentro de la parcela, el patrón espacial y el tamaño de las unidades muestrales. El tercer condicionante contribuye a reconocer el grado de uniformidad en la distribución de los individuos de cada especie. Es decir, aquellas especies que presentan un valor mayor son aquellos que poseen un patrón regular mientras aquellas con valor bajo son características de un patrón agregado, irregular y disperso.

Las especies con mayor densidad relativa son Baccharis salicifolia, Glandularia hookeriana y Larrea nítida. La densidad absoluta en la cual se basa la relativa es el resultado del cociente entre la cantidad de individuos de una misma especie y la superficie muestreada. La dominancia relativa tiene como base el conocimiento de la cobertura es decir, la proporción de terreno ocupada por la proyección perpendicular de las partes aéreas de los individuos de la especie considerada (Matteucci y Colma, 2002). Para cada especie se realizó un promedio de las muestras de valores de coberturas tomadas en las parcelas. Los resultados demuestran que Larrea divaricata y Larrea nítida (Figura 6) son las especies de mayor cobertura. La primera influenciada principalmente por el número de individuos del total del muestreo mientras que en las últimas dos, el valor de dominancia está influenciado por la cobertura.

Figura 6. Larrea divaricata (a) y Larrea nítida (b)

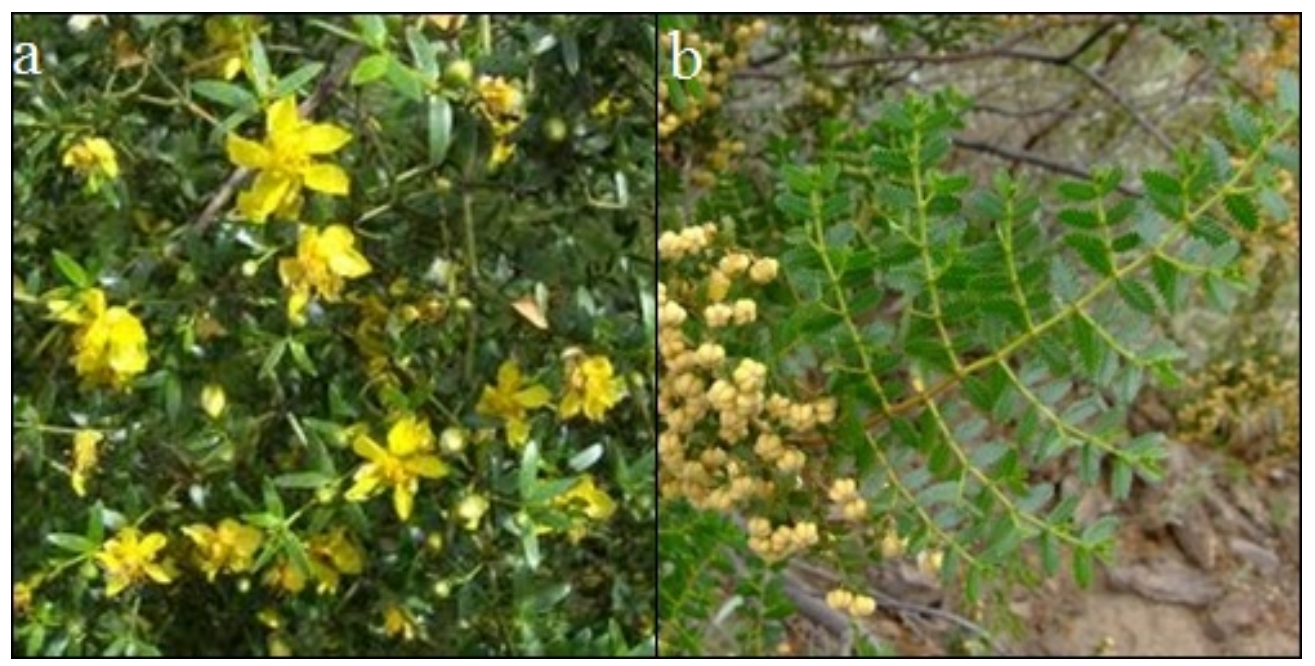

Fuente: fotografías de Renovales, 2013; Maipué, 2013. 
El índice de valor de importancia de cada especie se representa en la Figura 7. Las especies Larrea divaricata, Larrea nítida y Glandularia hookeriana poseen los valores más elevados por lo cual tienen una mayor importancia dentro de la comunidad florística muestreada. Entre otras especies importantes se puede mencionar a Baccharis salicifolia, Aloysia gratissima, Geoffroea decorticans, Condalia microphylla, Caesalpinia gilliesii. Este resultado muestra que la formación vegetal en esta zona es de Larrea divaricata y Larrea nítida.

Figura 7. Índice de importancia de cada especie.

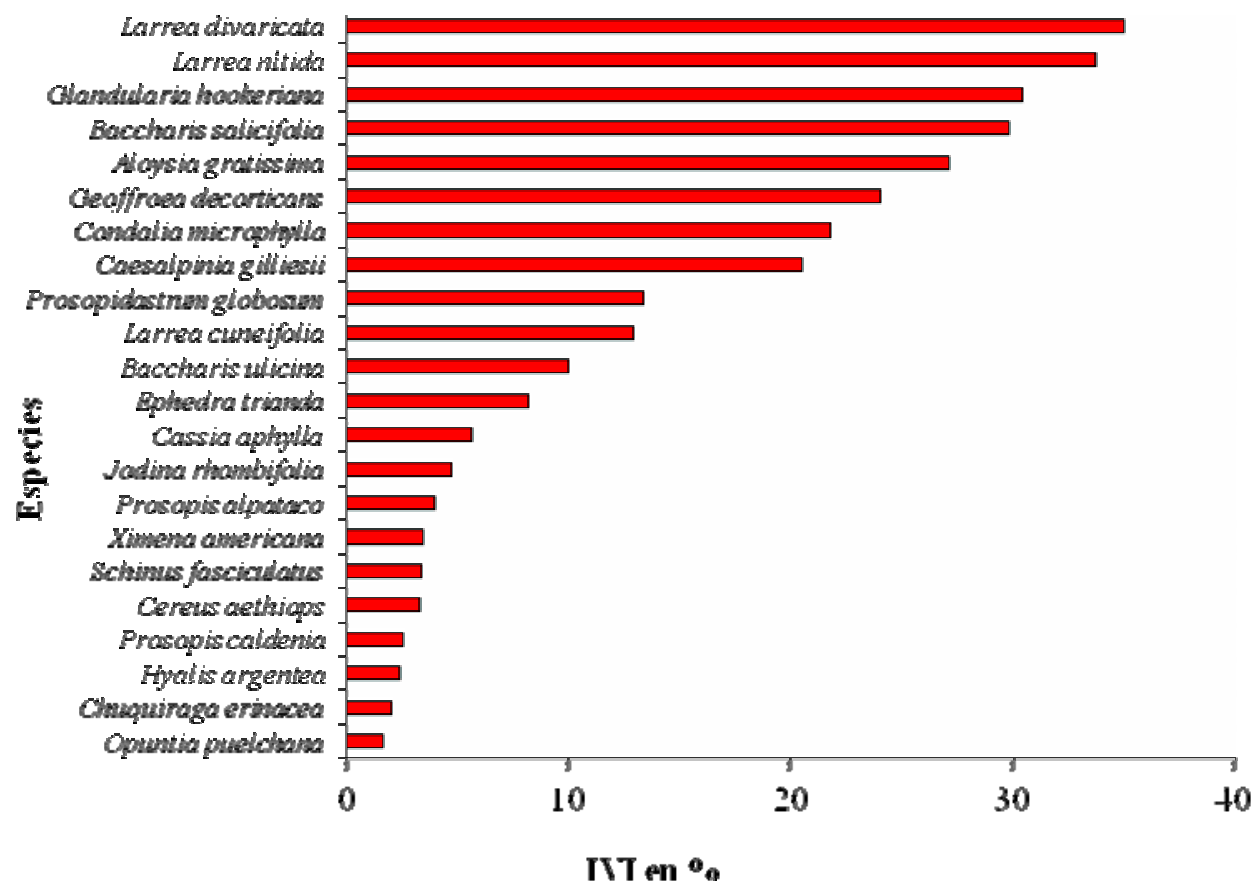

Fuente: Elaborado por Valeria S. Duval, 2013. 


\section{CONCLUSIONES}

El presente trabajo brindó información cuantitativa de la flora del jarillal en el Parque Nacional Lihué Calel. En las parcelas se relevaron un total de 796 individuos pertenecientes a 22 especies y a 9 familias. Presentó un predominio del biotopo arbustivo sobre el arbóreo. El primero estuvo representado por 19 especies y 722 individuos y el segundo biotopo por 3 especies y 74 individuos. Mediante la aplicación de los índices de diversidad y el valor de importancia se reconoció la relevancia del género Larrea en el monte. El índice de riqueza específica de Margalef fue de 7, 24 y Menhinick fue de 0,78, lo cual demostró que la vegetación posee una gran cantidad de especies. El índice de Simpson fue de 0,1038 lo cual demuestra que en la comunidad no existe una especie dominante, el índice de Shannon- Wiener con valor de 2,51 muestra que la comunidad es equitativa y el índice de equidad de Pielou fue de 0,86 por lo cual todas las especies son igualmente abundantes. Los valores de importancia definidos establecieron la formación vegetal del área como un monte de Larrea divaricata y Larrea cuneifolia. Esta formación vegetal de jarilla es diversa debido a que no presentó una especie dominante y la distribución es equitativa.

\section{BIBLIOGRAFÍA}

ÁlVAREZ, M., CÓRDOBA, S., ESCOBAR, F., FAGUA, G., GAST, F., MENDOZA, H., OSPINA, M., UMAÑA, A. M. y VILLAREAL, H. (2006). Manual de métodos para el desarrollo de inventarios de biodiversidad. Instituto de Investigación de Recursos Biológicos Alexander von Humboldt. Bogotá, Ramos López Editorial.

CANO, E. (2004). Inventario integrado de los recursos naturales de la provincia de la pampa. Clima, Geomorfología, Suelo y Vegetación. Buenos Aires, Instituto Nacional de Tecnología Agropecuaria provincia de La Pampa y Universidad Nacional de La Pampa.

COTTAM, G. y CURTIS, J.T. (1956). The use of distance measures in phytosociological sampling. Ecology, 37, 451- 460.

LUEBERT, F. y BECERRA, P. (1998). Representatividad vegetacional del Sistema Nacional de Áreas Silvestres Protegidas del Estado (Snaspe) en Chile. Ambiente y Desarrollo, 16 (2), $62-69$.

MARGALEF, R. (1969). El ecosistema pelágico del Mar Caribe. Memoria de la Sociedad de Ciencias Naturales La Salle, 29, 5-36.

MARGALEF, R. (1995). Ecología. Barcelona, Omega.

MATTEUCCI, S. (2012). Ecorregión Monte de Llanuras y Mesetas. En: Morello, J., Matteucci, S. y Colma, A. (2002): Metodología para el estudio de la vegetación. Buenos Aires, Ediciones Ave Fénix.

MATTEUCCI, S., RODRIGUEZ, A. y SILVA, M. (2012). Ecorregiones y complejos ecosistémicos argentinos. Buenos Aires, Orientación Gráfica Editora, 309-348. 
MAZZOLA, M., KIN, A., MORICI, E., BABINEC, F. y TAMBORINI, G. (2008). Efecto del gradiente altitudinal sobre la vegetación de las sierras de Lihué Calel (La Pampa, Argentina). Boletín Sociedad Argentina Botánica, 43, (1-2), 103-109.

MORELlO, J., MATTEUCCI, S., RODRIGUEZ, A. y SILVA, M. (2012). Ecorregiones y complejos ecosistémicos argentinos. Buenos Aires, Orientación Gráfica Editora.

MORENO, C. (2001). Métodos para medir la biodiversidad. Zaragoza, La Sociedad Entomológica Aragonesa.

MURGUÍA, M. y ROJAS, F. (2001). Biogeografía cuantitativa. Bousquets, J. y Morrone, J. (Ed.): Introducción a la biogeografía en Latinoamérica: teoría, conceptos, métodos y aplicaciones. México, Instituto de Ecología Ecosur, UNAM, 39- 47.

PASSERA, C., CAVAGNARO, B. y SARTOR, C. (2010). Plantas C3, C4 y CAM nativas

SMITH L.R. (2001). Ecología. Madrid, Pearson Educación, 62 - 64.

RAUNKAIER, C (1934): The life forms of plants and statistical plant geography. Oxford, Oxford University Press.

SUÁREZ, S. y VISCHI, N. (1997). Caracterización fisonómico-estructural de vegetación serrana (Alpa corral-Córdoba-Argentina). Multinequina, 6, 21-32.

UNIÓN INTERNACIONAL PARA LA CONSERVACIÓN DE LA NATURALEZA (2013). http://www.iucn.org/es/

ZABALZA, M. I., BARREIX, J. C. y CANO, E. (1989). Relevamiento fitosociológico del Parque Nacional Lihué Calel. Revista Facultad Agronomía Universidad Nacional La Pampa, 4 (1), 69-94. 